\title{
The optimization of the outputs of loose coupled SINS/GPS system
}

\author{
Yiting Liu a , Ying Xu ${ }^{b}$, Tingting $\mathrm{Li}^{\mathrm{c}}$, Xin Jin ${ }^{\mathrm{d}}$ \\ Science and Technology on Information Systems Engineering Laboratory, Nanjing 210007, China; \\ agcdlyt1985@163.com, bcele_xy@qq.com, corangeltt@163.com, dvenus_163@ccc.com
}

\begin{abstract}
In addition to check the availability of the Global Position System (GPS) receiver, residual $\chi 2$ detection method, which is used to assess the quality of auxiliary information provided by GPS and the fluctuations of the outputs of Kalman filter, is introduced in this paper. If the value of the detecting function is larger than the threshold value, the current navigation output minus the last navigation output and the difference is compared to another threshold which is set according to the motor performance of the vehicle. If the difference is bigger than the threshold, according to the change direction of the difference, the navigation results are corrected. While, if the difference is smaller than the threshold, the navigation results are output directly. The on-board experiments show that this method could monitor the fluctuations of outputs of GPS receiver and Kalman filter. And the precision of the attitude and velocity are greatly improved after the process of correction. The precision of the position is improved to a certain extent.
\end{abstract}

Keywords: Strapdown inertial navigation system (SINS); global position system (GPS); loose coupled assessment; correction.

\section{Introduction}

The strapdown inertial navigation systems (SINS) is widely used for the characters of not relying on outside information and providing the velocity, position and attitude of the vehicle in time. However, the accuracy of SINS mainly depends on the inertial measurement unit, gyros and accelerometers, and the system errors are accumulating over time, so SINS does not apply to long-endurance navigation [1]. While, the Global Position System (GPS) can provide the worldwide and all-weather navigation information, and its high positioning accuracy, speed measuring precision has nothing to do with time, but in the city or in the forests, the signal of GPS is easy to be affected by environment such as buildings or trees [2]. So, when SINS is combined with GPS effectively, the advantages of both will be complemented and the precision and performance of the integrated navigation system will be improved. The multi-sensor information fusion can make up for the limitations of single sensor measurement and increase the working reliability and stability of the integrated system [3-5]. However each sensor has its own using range and accuracy. During the measurement process, the sensors will be affected by measuring environment. Then, the output of each sensor contains disturbance and uncertainty which are caused by its principle and the external environment [6, 7]. These will inevitably influence the results of the information fusion. So, evaluating the outputs of sensors and judging whether the signals of sensors can be used is very important for improving information fusion result $[8,9]$. In this paper, one estimation method of GPS signal which is based on residual $\chi^{2}$ detection is proposed. According to the estimation result, one correction to the output of the SINS/GPS integration system is introduced too.

\section{Practical Problem and System Model}

In the loose coupled SINS/GPS system, Kalman filter is always used to fuse the navigation information of GPS and SINS, which could improve the accuracy and reliability of the navigation system. But sometimes the accuracy of such system is not as good as expectation because of the acquisition of GPS ephemeris signal is easily affected by the environment. Usually the GPS ephemeris signal becomes weak, then, the accuracy of GPS navigation information providing by GPS receiver will become worse. When the GPS signal is completely occluded, the ephemeris information will lost, at the same time, the GPS receiver will continue to output the last GPS navigation 
information before the GPS signal lost, and the ephemeris information flag changes. So, it can instantly stops the fusion of SINS and GPS according to the change of ephemeris information flag. When the GPS signal recovers, the fusion of SINS and GPS will recovered immediately. But at the beginning of the recovery, the output of Kalman filter oscillates and the accuracy of SINS/GPS integrated navigation system becomes worse because the one step prediction error covariance matrix of Kalman filter still retains the last state before the GPS signal lost. In addition, when the GPS signal becomes weak but does not lose completely, the precision of navigation information provided by the GPS receiver will become worse or even appears small oscillation. However, ephemeris signal flag will not change, and the integrated system will continue to fuse the information of SINS and GPS. Of course, the accuracy of the integrated navigation is affected. Figure 1 is the subtraction of the adjacent speed provided by GPS receiver in low dynamic environment. It can be seen that there are large errors in GPS navigation velocity when the signal is weakened.

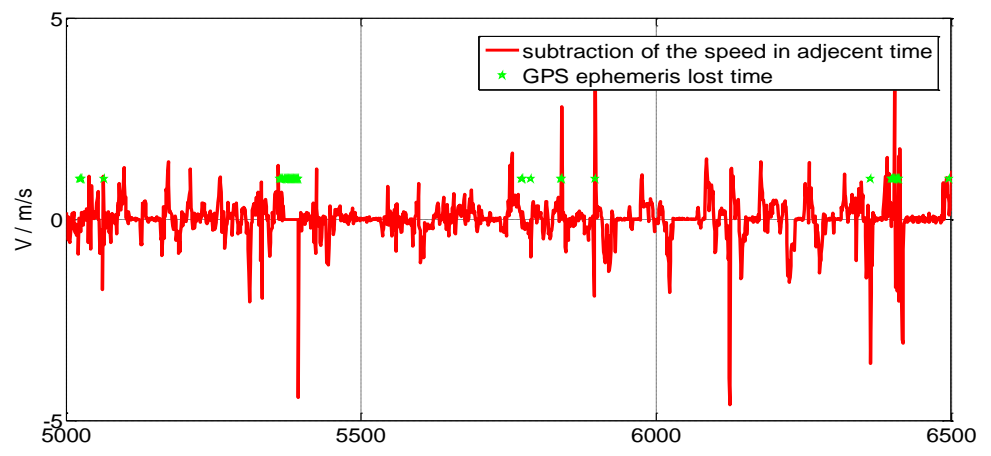

Fig. 1 Two or more references

According to the above two reasons which influence the precision of the loose coupled SINS/GPS integrated navigation system, one technology based on the auxiliary information evaluation and the outputs correction of loose coupled SINS/GPS is proposed in this paper. The schematic diagram is shown as Figure 2.

Where, $\boldsymbol{V}_{G P S}$ denote the velocities along the east, north and upward in geographic coordinate system provided by GPS. $\boldsymbol{V}_{\text {ins }}$ denote the velocities along the east, north and upward in navigation coordinate system provided by SINS. $\boldsymbol{P}_{G P S}$ are the latitude, longitude and height obtained by GPS. $\boldsymbol{P}_{i n s}$ are the latitude, longitude and height obtained by SINS.

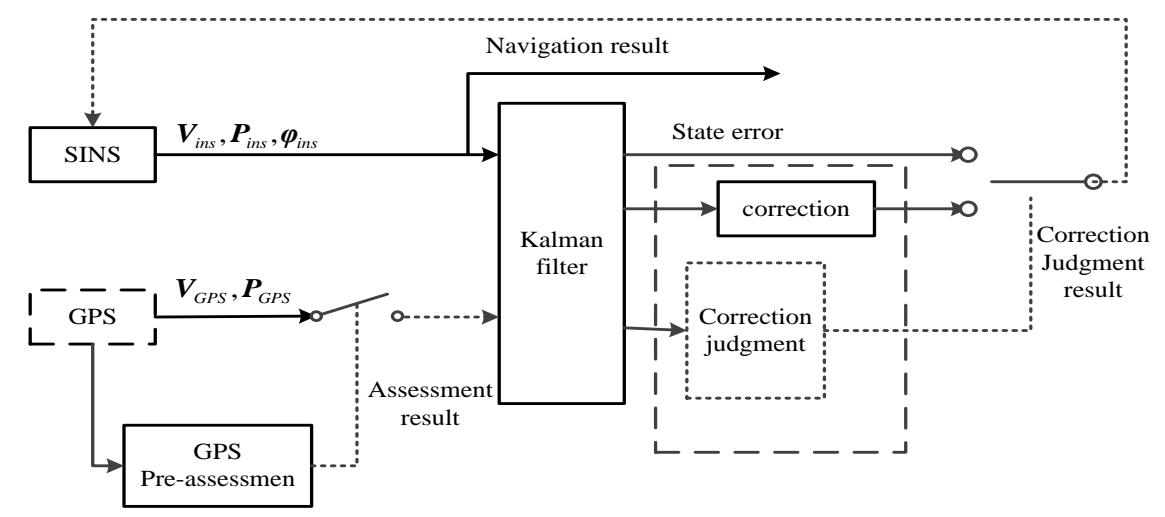

Fig. 2 The auxiliary information evaluation and the outputs correction of loose coupled SINS/GPS

From figure 2, we know that the GPS navigation information enters the GPS information pre-assessment module, firstly. Then, the GPS information pre-assessment module judges whether the current message is available according to ephemeris signal flag. If the GPS signal is unavailable, the information fusion is stopped immediately. If the GPS ephemeris information is available, whether the current GPS navigation information has fluctuations or its precision becomes lower should be judged by the correction judgment module. When the GPS information is not stable or its precision falls, the outputs of the integration is sentenced to be corrected. The correction module will modify the navigation results by the following introduced method. The modified attitude, velocity, position are the final navigation results. 
The discrete random linear model without controlling term of the SINS/GPS integrated navigation system is described as follows $[10,11]$.

$$
\left\{\begin{array}{l}
\hat{\boldsymbol{X}}_{k / k-1}=\phi_{k / k-1} \hat{\boldsymbol{X}}_{k-1}+\boldsymbol{W}_{k-1} \\
\boldsymbol{Z}_{k}=\boldsymbol{H}_{k} \hat{\boldsymbol{X}}_{k-1}+\boldsymbol{V}_{k-1}
\end{array}\right.
$$

Where, $\boldsymbol{X}=\left[\delta V_{E}, \delta V_{N}, \delta V_{U}, \delta \theta_{x}, \delta \theta_{y}, \delta \theta_{z}, \delta L, \delta \lambda, \delta h, \nabla_{x}, \nabla_{y}, \nabla_{z}, \varepsilon_{x}, \varepsilon_{y}, \varepsilon_{z}\right]^{T}$ denotes the state error vectors, $\delta V_{E}, \delta V_{N}, \delta V_{U}$ denote the east, north and upward velocity errors, respectively. $\delta \theta_{x}, \delta \theta_{y}, \delta \theta_{z}$ denote the pitch error, roll error and heading error. $\delta L, \delta \lambda, \delta h$ denote the latitude error , longitude error and the height error. $\nabla_{x}, \nabla_{y}, \nabla_{z}$ are the constant bias of the accelerometer in $\mathrm{x}, \mathrm{y}, \mathrm{z}$ axis. $\varepsilon_{x}$, $\varepsilon_{y}, \varepsilon_{z}$ are the constant bias of the gyros along $\mathrm{x}, \mathrm{y}, \mathrm{z}$ axes. $\phi_{k / k-1}$ denotes the one step state transition matrix form $k-1$ to $k . \boldsymbol{Z} \in \boldsymbol{R}^{6}$ denotes the measurement vectors, $\boldsymbol{H}$ is the measurement matrix, $\boldsymbol{W} \square N(0, \boldsymbol{Q})$ is the process noise, and $\boldsymbol{V} \square N(0, \boldsymbol{R})$ is the measurement noise.

\section{The Output Correction Judgment Method}

Although the residual $\chi^{2}$ detection method cannot determine the cause of the failure, it can determine the validity of a group of measurements in time, so the residual $\chi^{2}$ detection method is widely used in the mutant fault detection [12]. In this paper, it is used to judge whether the output of the SINS/GPS integrated navigation system need to be corrected.

In figure 2, the residual of the Kalman filter is

$$
\boldsymbol{r}_{k}=\boldsymbol{Z}_{k}-\boldsymbol{H}_{k} \hat{\boldsymbol{X}}_{k / k-1}
$$

Where $\hat{\boldsymbol{X}}_{k / k-1}$ is

$$
\hat{\boldsymbol{X}}_{k / k-1}=\phi_{k / k-1} \hat{\boldsymbol{X}}_{k-1}
$$

When the GPS signal is normal, the residual $\boldsymbol{r}_{k}$ of filter belongs to white noise. And its variance is

$$
\boldsymbol{A}_{k}=\boldsymbol{H}_{k} \boldsymbol{P}_{k / k-1} \boldsymbol{H}_{k}^{T}+\boldsymbol{R}_{k}
$$

Where, $\boldsymbol{R}_{k}$ is the variance matrix of the measurement noise, $\boldsymbol{P}_{k / k-1}$ is obtained from formula (5) to formula (7).

$$
\begin{aligned}
& \boldsymbol{P}_{k / k-1}=\phi_{k / k-1} \boldsymbol{P}_{k-1} \phi_{k / k-1}^{T}+\boldsymbol{Q}_{k-1} \\
& \boldsymbol{P}_{k-1}=\left(\boldsymbol{I}-\boldsymbol{K}_{k-1} \boldsymbol{H}_{k-1}\right) \boldsymbol{P}_{k-1 / k-2}^{T} \\
& \boldsymbol{K}_{k-1}=\boldsymbol{P}_{k-1} \boldsymbol{H}_{k-1}^{T} \boldsymbol{R}_{k-1}^{-1}
\end{aligned}
$$

When GPS signal loses, fluctuates or its precision reduces, the statistical characteristic of residual $\boldsymbol{r}_{k}$ changes, then, the mean of residual will no longer equal to 0 . So, the mean of residual can be used to judge whether the GPS signal is normal. The details of this method are as follows.

Firstly, a binary hypothesis to $\boldsymbol{r}_{k}$ is made as

$\mathrm{H}_{0}$ denotes no fault, where $E\left\{\boldsymbol{r}_{k}\right\}=0, E\left\{\boldsymbol{r}_{k} \boldsymbol{r}_{k}^{T}\right\}=\boldsymbol{A}_{k}$.

$\mathrm{H}_{1}$ denotes the existence of fault, where $E\left\{\boldsymbol{r}_{k}\right\}=\mu, E\left\{\left(\boldsymbol{r}_{k}-\mu\right)\left(\boldsymbol{r}_{k}-\mu\right)^{T}\right\}=\boldsymbol{A}_{k}$.

Then, the fault detection function is

$$
\boldsymbol{K}_{k-1}=\boldsymbol{P}_{k-1} \boldsymbol{H}_{k-1}^{T} \boldsymbol{R}_{k-1}^{-1}
$$

After the detection function is obtained, whether the system needs to be correction is determined according to the following criteria.

$\left\{\begin{array}{l}\text { if } \gamma_{k}>T_{D} \text {, correction } \\ \text { if } \gamma_{k} \leq T_{D} \text {, non-correction }\end{array}\right.$

Where, the threshold $T_{D}$ which determines the performance of the fault detection method. 


\section{The Output Correction of the Loose Coupled SINS/GPS System}

Generally, the accuracy of GPS information is very high in an open environment without any occlusion. But when passing through the city, tunnel or woods, the GPS signal will be weakened or interrupt because of those occlusion. Then, the position and the velocity accuracy reduce or be unavailable. And when GPS signal recovers, the output of the integrated system will oscillate which can also reduce the navigation precision during the early time because of the Kalman filter work again. In order to avoid these, the output of the SINS/GPS integration need to be corrected. The aim of the correction is that the velocity or position change rate of the loose coupled SINS/GPS integrated system should be less than the real required motor velocity and position change rate. The specific methods of correction are as follows [13-17].

(1) Initialize $\boldsymbol{P}_{\text {out }}(k), \boldsymbol{V}_{\text {out }}(k)$ with $\boldsymbol{0}$.

(2) The filter working stability and the GPS signal is stable

When the GPS signal is stable and the filter works in a stable stage, then, the value of the detection function obtained from the correction judgment module is less than the threshold $\gamma_{k}>T_{D}$. So, it can be concluded that the GPS navigation information is normal which can be used directly. Then

$$
\begin{aligned}
& \boldsymbol{P}_{\text {out }}(k)=\boldsymbol{P}_{\text {ins }}(k)-\tilde{X}_{P}(k) \\
& \boldsymbol{V}_{\text {out }}(k)=\boldsymbol{V}_{\text {ins }}(k)-\tilde{\boldsymbol{X}}_{V}(k)
\end{aligned}
$$

Where, $\tilde{\boldsymbol{X}}_{p}(k)=\boldsymbol{X}_{p}(k), \tilde{\boldsymbol{X}}_{V}(k)=\boldsymbol{X}_{V}(k)$, which means that the correction to the position and velocity obtained from SINS are equal to the estimation of the Kalman filter. $\boldsymbol{X}_{p}(k)=\left[\begin{array}{lll}\delta L, & \delta \lambda, \quad \delta h\end{array}\right]$, $\boldsymbol{X}_{V}(k)=\left[\delta V_{E}, \quad \delta V_{N}, \quad \delta V_{U}\right]$ are the position and velocity error estimation of the Kalman filter.

(3) At he begin of the filter working stage or the GPS navigation information fluctuated

At the begin of the filter working stage or the GPS signal become weak, the value of the detection function obtained from the correction judgment module is bigger than the threshold $\gamma_{k}>T_{D}$. Then, the position and the velocity of SINS/GPS integrated navigation results should be corrected in 3 kinds of circumstances.

A) When $\boldsymbol{P}_{\text {out }}(k)-\boldsymbol{P}_{\text {out }}(k-1) \geq \Delta \boldsymbol{P}_{D}$, the correction method is

$$
\boldsymbol{P}_{\text {out }}(k)=\boldsymbol{P}_{\text {out }}(k-1)+\Delta \boldsymbol{P}\left[T_{n}\right.
$$

Where, $\Delta \boldsymbol{P}_{D}$ is the position change rate of the adjacent filter correction moments, which is the second criterion used to correct the position. The value of the $\Delta \boldsymbol{P}_{D}$ should be conform to the actual motor character of the carrier. $T_{n}$ is the filter cycle.

In the same way, when $\boldsymbol{V}_{\text {out }}(k)-\boldsymbol{V}_{\text {out }}(k-1) \geq \Delta \boldsymbol{V}_{D}$, the velocity correction method is

$$
\boldsymbol{V}_{\text {out }}(k)=\boldsymbol{V}_{\text {out }}(k-1)+\Delta \boldsymbol{V} \square T_{n}
$$

Where, $\Delta \boldsymbol{V}_{D}$ is the velocity change rate of the adjacent filter correction moments, which is the second criterion used to correct the velocity. The value of $\Delta \boldsymbol{V}_{D}$ is always very small in the low dynamic environment. $T_{n}$ is the filter cycle.

B) When $\boldsymbol{P}_{\text {out }}(k)-\boldsymbol{P}_{\text {out }}(k-1) \leq-\Delta \boldsymbol{P}_{D}, \boldsymbol{V}_{\text {out }}(k)-\boldsymbol{V}_{\text {out }}(k-1) \leq-\Delta \boldsymbol{V}_{D}$, the correction details are

$$
\begin{aligned}
& \boldsymbol{P}_{\text {out }}(k)=\boldsymbol{P}_{\text {out }}(k-1)-\Delta \boldsymbol{P} \llbracket T_{n} \\
& \boldsymbol{V}_{\text {out }}(k)=\boldsymbol{V}_{\text {out }}(k-1)-\Delta \boldsymbol{V} \boldsymbol{T}_{n}
\end{aligned}
$$

C) When $\left|\boldsymbol{P}_{\text {out }}(k)-\boldsymbol{P}_{\text {out }}(k-1)\right|<\Delta \boldsymbol{P}_{D},\left|\boldsymbol{V}_{\text {out }}(k)-\boldsymbol{V}_{\text {out }}(k-1)\right|<\Delta \boldsymbol{V}_{D}$, the correction method is

$$
\begin{aligned}
& \boldsymbol{P}_{\text {out }}(k)=\boldsymbol{P}_{\text {ins }}(k)-\tilde{\boldsymbol{X}}_{p}(k) \\
& \boldsymbol{V}_{\text {out }}(k)=\boldsymbol{V}_{\text {ins }}(k)-\tilde{\boldsymbol{X}}_{V}(k)
\end{aligned}
$$




\section{Testing in Vehicle Environment}

The auxiliary information evaluation and the output correction method is tested by the vehicle experiment. A prototype of fiber optic gyroscope strapdown inertial navigation system is used in the experiment. The gyro constant drift is $0.006 \%$, and its random drift is $0.005 \% \sqrt{\mathrm{h}}$, the accelerometer constant bias is less than $50 \mu \mathrm{g}$. The GPS information is obtained by FlexPark6 GNSS receiver developed by NovAtel firm. The filter cycle is $1 \mathrm{~s}$. The reference navigation data comes from the loose couple of PHINS developed by a French firm, IXBLUE, and the FlexPark6 GNSS receiver. PHINS and inertial measurement unit are fixed on the same mounting plate shown in Fig. 3. Moreover, Fig. 4 is the navigation experimental car with a red circle in the Fig. 4 to mark the GNSS receiver antenna. In the experiment, the experimental car is drove normally, which was drove past the tall buildings, woods and tunnel.

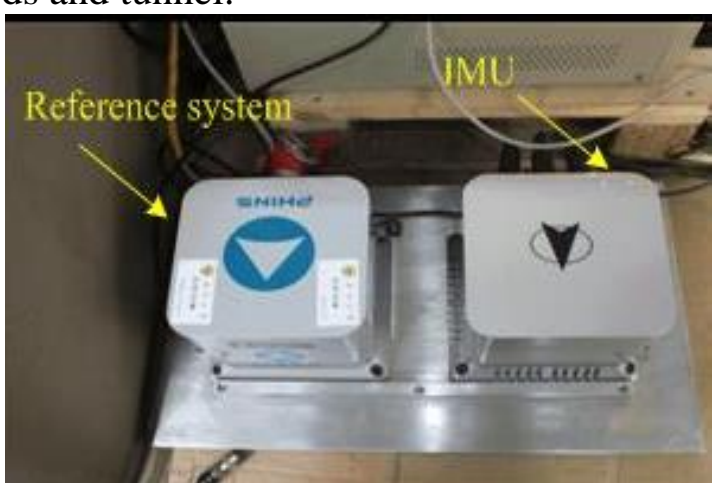

Fig. 3 Installation diagram

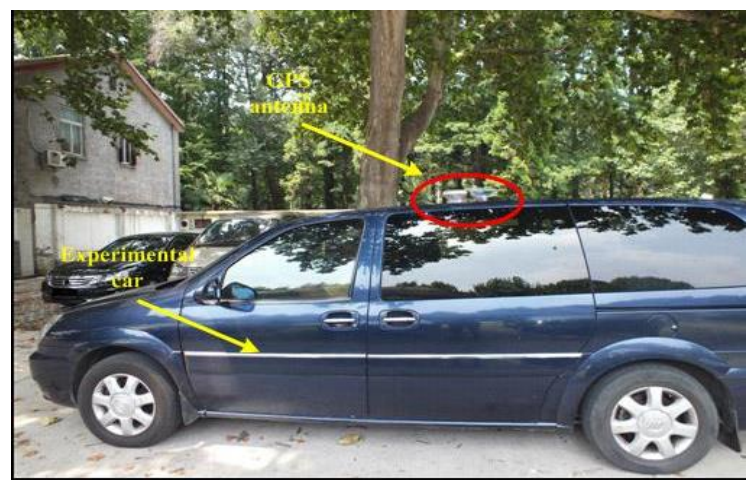

Fig. 4 Experimental car

Figure 5, figure 6, figure 7 are the attitude error curves, velocity error curves and the position error curves of the loose coupled SINS/GPS, where the blue dotted line is the result of the system which only stops information fusing when the GPS signal is lost. While, the red solid line denotes the integrated result is corrected by the method proposed in this paper.

From Figure 5(a), the attitude errors of SINS/GPS integrated navigation system fluctuant with large amplitude only by judging whether the available satellites are normal. The maximum values of pitch error, roll error and heading error are $3.585^{\circ}, 3.615^{\circ}$ and $9.81^{\circ}$. While, after the assessment and correction steps, which results are shown in Figure 5(b), the horizontal attitude errors are within $0.1^{\circ}$, and the heading error is $-0.163^{\circ}$ after $8000 \mathrm{~s}$. The attitude accuracy of integrated navigation system has greatly improved by the assessment and correction. The statistical results of the attitude errors are shown in Table 1.

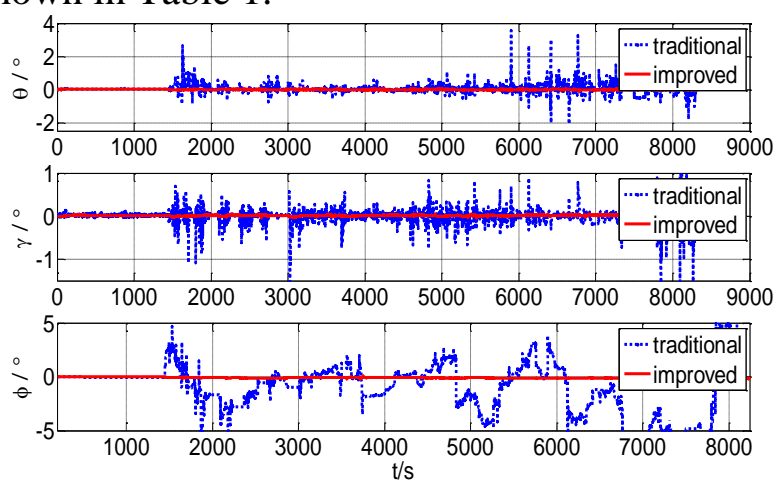

(a) Attitude error curve

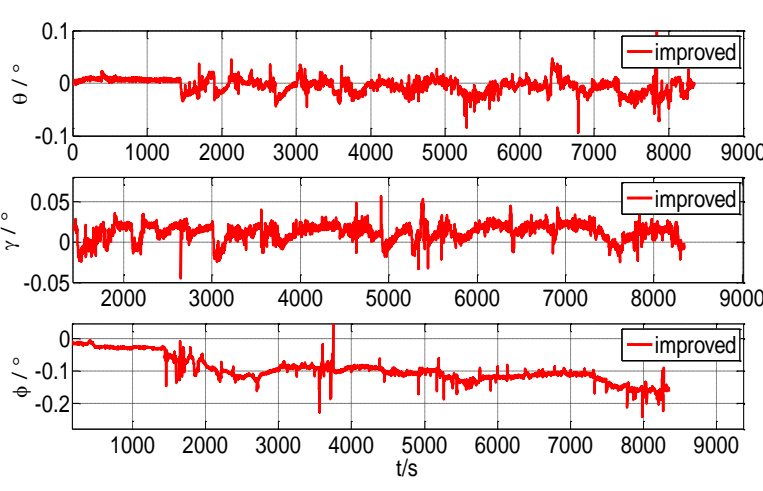

(b) The local amplification figure

Fig. 5 The attitude error curves of the loosely coupled SINS/GPS integrated navigation system 
Table 1 The statistics of the attitude error before and after improvement (Unit: ०)

\begin{tabular}{ccccccc}
\hline & \multicolumn{2}{c}{ pitch } & \multicolumn{2}{c}{ roll } & \multicolumn{2}{c}{ heading } \\
\cline { 2 - 7 } & mean & SD & mean & SD & mean & SD \\
\hline Before improvement & 0.0220 & 0.274 & 0.0156 & 0.184 & -0.232 & 2.92 \\
After improvement & -0.00258 & 0.0135 & 0.0149 & 0.00972 & -0.103 & 0.0403 \\
\hline
\end{tabular}

Where, SD means standard deviation.

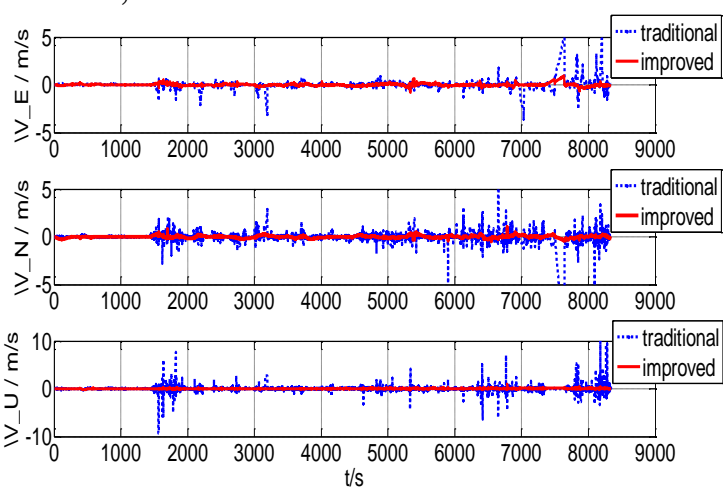

(a) Speed error curves
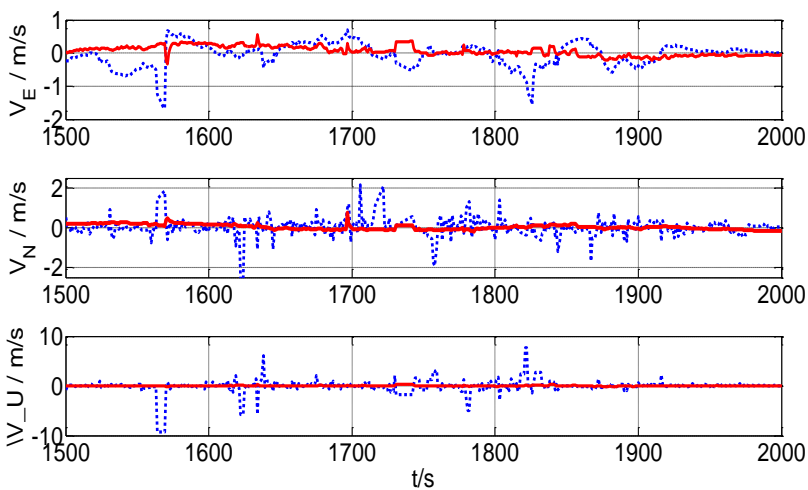

(b) The local amplification figure

Fig. 6 The speed error curves of the loosely coupled SINS/GPS integrated navigation system

From Figure 6(a), the velocity errors of the integrated navigation mixed with lots of burr only by judging whether the available satellites are available. And the maximum error values of east velocity, north velocity and upward velocity are $5.148 \mathrm{~m} / \mathrm{s}, 5.58 \mathrm{~m} / \mathrm{s}$ and $9.215 \mathrm{~m} / \mathrm{s}$. From Figure 6(b), the proposed assessment and correction methods have greatly improved the velocity accuracy of the SINS/GPS integrated navigation, the velocity errors are within $1 \mathrm{~m} / \mathrm{s}$. The statistical results of the velocity errors are shown in Table 2.

Table 2 The statistics of the speed error before and after improvement

\begin{tabular}{ccccccc}
\hline & \multicolumn{2}{c}{$\mathrm{V}_{\mathrm{E}} / \mathrm{m} / \mathrm{s}$} & \multicolumn{2}{c}{$\mathrm{V}_{\mathrm{N}} / \mathrm{m} / \mathrm{s}$} & \multicolumn{2}{c}{$\mathrm{V}_{\mathrm{U}} / \mathrm{m} / \mathrm{s}$} \\
\cline { 2 - 7 } & mean & $\mathrm{SD}$ & mean & $\mathrm{SD}$ & mean & $\mathrm{SD}$ \\
\hline Before improvement & 0.04759 & 0.6 & -0.105 & 0.969 & 0.04236 & 0.670 \\
After improvement & 0.00967 & 0.142 & -0.00330 & 0.122 & 0.02286 & $0 . .0609$ \\
\hline
\end{tabular}

From figure 7(a) and Figure 7(b), it can be conclude that most of the large burr in position errors have been eliminated. The proposed assessment and correction methods have improve the position accuracy of the SINS/GPS integrated navigation to a certain extent. The statistical results of the velocity errors are shown in Table 3.

Table 3 The statistics of the position error before and after improvement

\begin{tabular}{ccccccc}
\hline & \multicolumn{2}{c}{ latitude/ } & \multicolumn{2}{c}{ Longitude/ } & \multicolumn{2}{c}{ height $/ \mathrm{m}$} \\
\cline { 2 - 7 } & mean & SD & mean & SD & mean & SD \\
\hline Before improvement & $-5.277 \mathrm{e}-5$ & 0.0004631 & $3.014 \mathrm{e}-5$ & 0.000303 & 0.7915 & 5.97 \\
After improvement & $-2.224 \mathrm{e}-6$ & $9.027 \mathrm{e}-5$ & $1.453 \mathrm{e}-5$ & $9.771 \mathrm{e}-5$ & 0.4978 & 5.309 \\
\hline
\end{tabular}



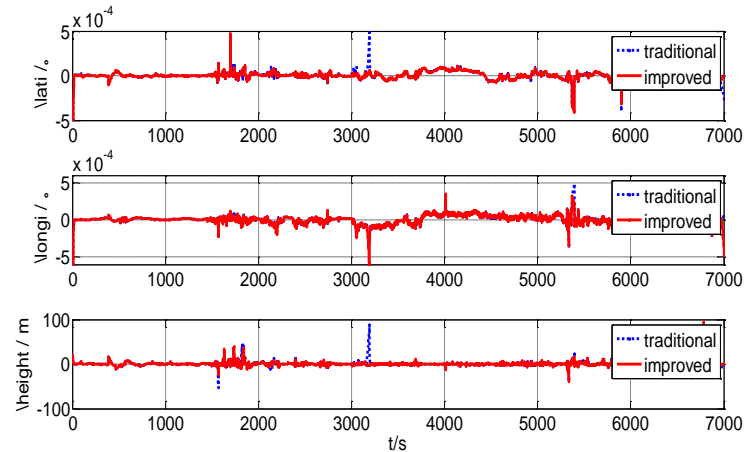

(a) Position error curves
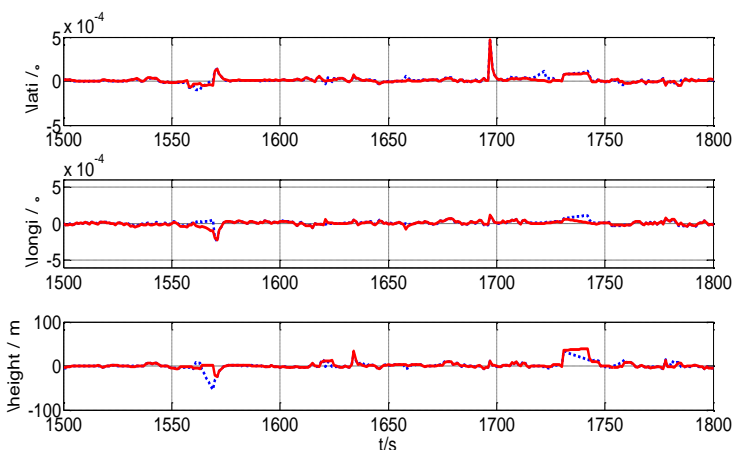

(b) The local amplification figure

Fig. 7 The position error curves of the loosely coupled SINS/GPS integrated navigation system

\section{Summary}

In this paper, the residual $\chi^{2}$ detection method is introduced in the SINS/GPS loose coupled system to evaluate GPS signal in real time. The results of the vehicle experiments show that the value of residual $\chi^{2}$ square detection function changes when the GPS signal being sheltered but not completely unavailable, oscillating or the oscillation of Kalman filter at the beginning of GPS signal recovery. Comprehensively considering the evaluating the result and comparing the subtraction of navigation results in the adjacent filter cycle and the actual motor vehicle performance, we can greatly improve the attitude and velocity accuracy of the loose coupled SINS/GPS system and improve the position accuracy to a certain extent by the output correction method to the navigation results. The evaluation method of GPS information and the output correction scheme proposed in this paper are simple and easy to realize which has a certain practicability in engineering.

\section{References}

[1] Yuanxin Wu, Jinling Wang, Dewen Wu. A new technique for INS/GNSS attitude and parameter estimation using online optimization. IEEE TRANSACTIONS ON SIGNAL PROCESSING. Vol. 62 (2014) No. 10, p. 2642-2655.

[2] Xiaolin Gong, Wei Fan, Jiancheng Fang. Application of unscented R-T-S smoothing based on novel mathematical model in SINS/GPS integrated system post processing. Measurement. Vol. 55 (2014), p.581-592.

[3] Tian Xueyi. The technology research of multi-sensor data association and track fusion. A Dissertation for the Degree of D. Engineer, Harbin Engineering University, China, 2012, p. $10-24$

[4] Katia Sycara, Robin Glinton, Binyu. An integrated approach to high-level information fusion. Information fusion. Vol. 10 (2009), p.25-50.

[5] Petrovic V.S., Xydeas C.S. Gradient-based multi-resolution image fusion. IEEE Transactions on Image Processing. Vol. 13 (2004) Nol.2, p.228-237.

[6] Oberkampf W L, Helton J C, and Joslyn C A, et al. Challenge problems: uncertainly in system response given uncertain parameter. Reliability Engineering \& System Safety. Vol. 85(2004), No.1-3, p.11-19.

[7] Smith D, Singh S. Approaches to multisensory data fusion in target tracking: a survey. IEEE Transactions on Knowledge and Data Engineering. Vol. 18 (2006) No.12, p.1696-1710.

[8] Nassar S., EI-Sheimy. A Combined Algorithm of Improving INS Error Modeling and Sensor Measurements for Accurate INS/GPS Navigation. GPS Solute. Vol. 10 (2006), p.29-39. 
[9] X.L. Gong, R. Zhang. Analysis the effect of IMU calibration errors on SINS/GPS integration accuracy for airborne earth observation. Proceedings of the 3rd international Symposium on information Engineering and Electronic Commerce.2011: 38-42.

[10] Tao Zhang, Xiaosu Xu. A new method of seamless land navigation for GPS/INS integrated system. Measurement. Vol. 45 (2012), p.691-701.

[11]Wang Xinlong, Li Yafeng. An innovative scheme for SINS/GPS ultra-tight integration system with low-grade IMU. Aerospace Science and Technology. Vol. 23 (2012), p. 452-460.

[12] Qin Yongyuan, Zhang Hongyue, Wang Shuhua. The principles of Kalman filter and integrated navigation. Northwesten Polytechnical university Press, 2012, p.245-250.

[13]Qian Huaming. Research on fault diagnosis and tolerant technology and its application to integrated navigation system. A Dissertation for the Degree of D. Engineer, Harbin Engineering University, China, 2004, p.43-59.

[14]Zheng Xin, Fu Mengyin, Zhou Zhanghua. Smoothing processing method of local reference output speed in response to master reference speed error jump. Journal of Chinese Inertial Technology. Vol. 20 (2012) No.1, p.24-28.

[15] Stancic R, Gravac S. The integration of strapdown INS and GPS based on adaptive error damping. Robotics and Autonomous System. Vol. 58 (2010) No.10, p.1117-1129.

[16]Zhao Min, Zhao Liping. Anti-jamming Technologies for Global Satellite Navigation and Position System. Command Information System and Technology. Vol. 7 (2016) No. 7, p. 37-40.

[17] Wang Ke, Hui Xincheng, Guo Zhiqiang, et. al. Multi-platform Sensor Registration Method in Geodetic Coordinates for Naval Formations. Command Information System and Technology. Vol. 4 (2013) No. 5, p. 71-75. 